\title{
Antioxidants in the retina and vitreous - current state of knowledge
}

\author{
Agata Pietras-Baczewska $\circledR^{1}$, Katarzyna Nowomiejska ${ }^{\circledR}{ }^{1}$, Małgorzata Sztanke $\mathbb{D}^{2}$, \\ Mario Damiano Toro ${ }^{1}{ }^{1,3}$, Robert Rejdak (1) \\ 'Department of General Ophthalmology, Medical University of Lublin, Lublin, Poland \\ ${ }^{2}$ Chair and Department of Medical Chemistry, Medical University of Lublin, Lublin, Poland \\ ${ }^{3}$ Faculty of Medicine, Collegium Medicum Cardinal Stefan Wyszynski University, Warsaw, Poland
}

\begin{abstract}
In the healthy organism, there is a constant balance between the formation and neutralization of free radicals. Oxidative stress is a result of free radicals' production and naturalization imbalance, in favor of the free radicals' high concentration. Literature suggests the existence of the relationship between decreased intraocular antioxidant capacity and ocular diseases. Retina and the photoreceptors in particularl, are susceptible to oxygen deficiency due to their great oxygen consumption. The aim of this review was to describe the relationship between oxidative stress and the most common vitreoretinal disorders. The authors focused on four ocular diseases such as vitreous degeneration, rhegmatogenous retinal detachment, age-related macular degeneration and diabetic retinopathy. It was widely proven that high oxidative stress damages retina by the acceleration of photoreceptors and ganglion cells apoptosis. Available data suggesting that substances scavenging oxidative stress may be effective in slowing down the progression of these degenerative ocular diseases. However, the effects of antioxidants treatment are ambiguous, successful results of experimental studies lead straight to clinical use in human in the future.
\end{abstract}

KEY WORDS: antioxidants; antioxidant capacity; oxidative stress; vitreous degeneration; retinal detachment; diabetic retinopathy; age-related macular degeneration

Ophthalmol J 2020; Vol. 5, 81-86

\section{INTRODUCTION}

Oxygen is the essential vital chemical element. It can be either consumed by the cells remaining in its molecular form or be transformed into insoluble reactive oxygen species (ROS). Reactive oxygen derivatives such as superoxide aions (O2-), hydrogen peroxide (H2O2), hydroxyl radical $(\mathrm{OH}-)$, peroxyl radical and singlet oxygen are the most common radicals in the organisms [1]. Free radicals are the molecules containing unpaired electrons making them capable of independent existence. The unpaired electron makes the molecules highly reactive. They can either donate an electron or extract an electron from other molecules, behaving as a reductant or oxidant.
Vivid reactivity of radicals is the cause of their short half-life (10-4 seconds or less) in any biological environment [2]. In the healthy organism, there is a constant balance between the formation and neutralization of free radicals. Free radicals' formation is a natural consequence of cellular functioning, but one has to remember that exogenous factors can trigger radicals' overproduction. Any substance or molecule with the capacity of delaying or inhibiting oxidation of other substance is generally defined as an antioxidant. There are different types of antioxidants, they can be both exogenous and endogenous, but they are all responsible for the prevention of radicals' induced cell damage and death. All antioxi- 
dants can by divided into three main groups - antioxidant enzymes, chain-breaking antioxidants and metal bonding proteins [2].

Oxidative stress is a result of free radicals' production and naturalization imbalance, in favor of the free radicals' high concentration. The imbalance can be either due to overproduction of endogenous ROS by mitochondrial respiration or decreased antioxidant capacity [3]. ROS damage range of molecular elements including proteins, lipids and nucleic acids (DNA and/or RNA). These cellular structures destruction contributes to cell aging, acceleration of genetically programmed cell death or apoptosis [1]. There are suggestions in the literature concerning the relationship between decreased intraocular antioxidant capacity and the ocular diseases. Several age-related ocular diseases have been described as associated with oxidative stress, such as Fuch's dystrophy, nuclear cataract, age-related macular degeneration (AMD), diabetic retinopathy (DR) [4].

The aim of this review was to describe the relationship between oxidative stress and vitreoretinal disorders.

\section{VITREOUS DEGENERATION}

Vitreous body is the biggest structural component of the eye, being roughly $80 \%$ of the total globe size. Vitreous cavity is the space between the posterior lens surface and the inner limiting membrane (ILM) of the retina. Vitreous has approximately $4 \mathrm{ml}$ of volume and it is built of water $(99 \%)$, collagen fibres, non-collagen proteins, glucosaminoglycans (e.g. hyaluronic acid) [5]. It is relatively acellular with only one layer of mononuclear phagocytes and hyalocytes in the posterior part of its cortex [6]. Due to its clarity vitreous enables the photons to freely penetrate to posterior eye elements, such as retina. It also takes part in maintaining intraocular pressure and intraocular oxygen tension. As vitreous body is an avascular structure it has no capacity of regeneration once it is damaged [7].

Vitreous degeneration is a physiological age-related process resulting from gel liquefaction and concurrent dehiscence of vitreoretinal adhesion [8]. As the gel liquefies the adhesion of vitreal body to retina weakens. In consequence vitreal cortex separates totally form the ILM of the retina, which literature refers as posterior vitreous detachment (PVD). Although it is said that vitreous degeneration is a physiological phenomemnon, there are factors which can facilitate the process. Increased oxidative stress biomarkers and increased activity of proteolytic enzymes are said to be positively correlated with the vitreous degeneration [9]. A high concentration of ROS induced by light was presented as the liquefication factor to be age-related by Uneo and collegues [10]. It was also proven that free radicals, such as hydroxyl radical $(\mathrm{OH}-)$ and superoxide anion (02-), generated by visible light irradiation, contribute to vitreous liquefaction [11]. Berra and associates found that the antioxidative potential of human vitreous decrease with age which contribute to subsequent oxidative-stress-induced retinal pathologies [12].

\section{RETINAL DETACHMENT}

Rhegmatogenous retinal detachment (RRD) is one of the ophthalmological emergencies. The epidemiology of RRD varies. Different studies show there is 1 person per 10.000 yearly [13] while others estimate the incidence of approximately 6-18 cases for every 100.000 people a year [14]. Following the definition retinal detachment occurs when the neurosensory retina (NSR) separates from the retinal pigment epithelium (RPE). There are four types of retinal detachment distinguished on the basis of the underlying cause. The majority of RRD cases derives from the retinal break which becomes an entrance to subretinal space. Leakage of the vitreous cavity fluid leads to separation of the NSR [15]. Detached sensory retina is deprived of oxygen and nutrients, thus leading to stress conations and ROS overproduction in photoreceptors [16]. Both photoreceptors and RPE are very susceptible to ROS formation due to their high proportion of fatty acids, high oxygen consumption and permanent exposure to sunlight.

Study shows that biological antioxidative potential (BAP) was significantly deceased in the vitreous fluid of patients with RRD. Oxidative stress was elevated as the result of retinal cells damage. What is more, authors imply that the level of BAP seems to be correlated only with the extent of retinal detachment, with no proved correlation with duration, presence of proliferative vitreoretinopathy (PVD), vitreous hemorrhage (HV), macular status or patients' age [17]. Photoreceptors are very susceptible to oxygen deficiency due to their great oxygen consumption. They have a large number of mitochondria to fulfil their metabolic demand. A detachment of neurosensory retina form under- 
lying retinal segments leads to hypoxic stress and other nutrients deprivation. The exact mechanism of cell death is not yet clearly known. It was found that oxidative stress causes mitochondrial and extra-mitochondrial aerobic ATP synthesis leading to ROS overproduction. Elevated ROS concentration, however, is proven to accelerate cell apoptosis. The experimental study questioned whether decrease of oxidative stress would have an effect on mitochondrial dynamics in photoreceptors after retinal detachment. The outcomes showed inhibition of essential mitochondrial fission mediator and decreased photoreceptor death [18]. A different study, conducted on the experimental retinal detachment, proved successfull photoreceptors protection by antioxidative substances as edaravone or tauroursodeoxycholic acid [19]. Another antioxidant factor - N-acetylocysteine (NAC) was specifically examined for its possible use in preventing retinal detachment, secondary do PVR. N-acetylcysteine, a ROS scavenger, was introduced subretinally at the time of $\mathrm{RD}$ induction. The experiment was conducted on rabbit eyes and cell models. The outcome showed that $\mathrm{N}$-acetylcysteine (NAC) effectively decreased the accumulation of ROS. Although NAC therapy prevented rabbits from retinal detachment itself, the majority of the animals treated still developed PVR membranes. This study makes one consider the future therapeutic use and clinical application of $\mathrm{N}$-acetylocysteine in prevention of RD [20].

\section{AGE-RELATED MACULAR DEGENERATION}

Age-related macular degeneration (AMD) is listed as one of the XXI-century diseases [21]. It is macular pathology of the elderly. Its incidence increases with age however it usually does not affect people before 50 years of age. The course of the disease may have different rates of development. Any type causes irreversible visual impairment, gradually leading to blindness if left untreated. Researchers estimate that the number of individuals with AMD to be 196 million in 2020 with the tendency to increase to 288 million in 2040 [22]. AMD is generally characterized as drusen, pathologies of pigment epithelium (RPE) or pathological vessels formation in the central retina. Literature classifies AMD into two types - "dry" and "wet". The first type called 'dry-type AMD' is the great majority of cases, being approximately $90 \%$ of the population suffering from AMD. It is described as the formation of drusen - deposits of metabo- lism products localized between RPE and Bruch's membrane. RPE is very susceptible to ROS influence mostly due to its high oxygen consumption and exposure to light. As the AMD progresses, the degeneration of RPE spreads through resulting in "geographical atrophy" (GA), well visible in fundus examination. Approximately $10-15 \%$ of patients suffer from "wet-type AMD". Vision impairment in this AMD type is greater and the course of the disease is more rapid in comparison to dry-type. Clinically it is characterized as the choroidal neovascularization $(\mathrm{CNV})$ or pigment epithelium detachment (PED). Newly formed sub-RPE vessels are very weak which leads to vascular leakages or hemorrhage causing further retina destruction and vision loss [23]. The only advantage of the rapidly progressing wet-type AMD over the dry-type AMD is the treatment method. Currently, there is no targeted treatment for early-stage drusen formation nor advanced geographic atrophy in dry AMD. On the contrary, patients suffering from wet AMD can be successfully treated with intraocular anti-VEGF injections (anti-vascular endothelial growth factor). It has been widely proven that antioxidant activity decreases with age. Moreover, the level of ROS increases, and thus the total antioxidant capacity of any tissue [24]. Age-related macular degeneration is still being tested for genetical inheritance. Studies had shown individuals with the complement factor $\mathrm{H}(\mathrm{CFH})$ polymorphism of $\mathrm{Y} 402 \mathrm{H}$ are at greater risk of suffering from AMD. This type of polymorphism reduces the CHF's ability to neutralize the effect of oxidized photoreceptor phospholipids, which later triggers RPE cells' apoptosis [25]. Retinal pigment epithelium damage is observed even in the early stages of AMD, however, the correlation between the damage extent with the OS and disease progression has not been found [26]. Retinal pigment epithelium cells are known to have high metabolic activity. The two-compound pigment of macula (zeaxantin and lutein) is the barrier, which protects the cells form oxidative stress [27]. The cells themselves have their own antioxidant system. The NRF-2/ARE (nuclear factor-erythroid 2-related factor-2/antioxidant response element) complex plays great role in the oxidative stress regulation [28]. In the oxidative stress environment, the NRF-2 has released the complex and is tan moved from cytostol to cell nucleus allowing antioxidant system to be activated [29]. The experimental study on mice models, proved that the loos of NRF-2 gene caused more intensive oxidative stress and what is more the 
RPE became more susceptible to oxidative damage [30]. On the contrary to inheritance, AMD RPE damage can be also caused by environmental factors. These modifiable external factors; they are cigarettes smoking or high fat consumption. The exact correlation between cigarette smoking and AMD progression is not yet clearly known. However, data shows substances from cigarette smoke diminish tissues from ascorbic acid and protein sulphyrdyl groups, leading to DNA, lipids and proteins oxidation and damage.

The molecular changes, like these caused by cigarette smoke, have been described in AMD. This fact could lead to assumption that oxidative damage is one of the factors in the mechanism of AMD disease development or progression. The same observations had been made in the multiple studies concluding that smoking is the strongest environmental risk factor for AMD [31].

\section{DIABETIC RETINOPATHY}

Diabetes is a major cause of secondary blindness in developed countries in people of working age. It is a civilization disease and a great epidemiological problem. It is estimated that over 420 million people with diabetes currently live in the world. World Health Organisation predicts that diabetes will become the seventh death cause by 2030 [32]. The vast majority of diabetics, reaching even $90 \%$, are people with type 2 diabetes. It is now believed that in the next 15-20 years the number of diabetics in Poland will double. All patients with diagnosed diabetes and those with pre-diabetes are at risk of complications. The most important concern blood vessels (angiopathies) in the kidneys, brain, heart and eyes. Diabetic retinopathy is a complication associated with retinal vascular remodelling resulting from chronic hyperglycemia. Unfavorably for the patients, complications occur regardless of the type of diabetes. On the other hand, numerous studies had confirmed that the duration of the underlying disease has the greatest impact on the development of retinopathy. Treatment of complications is often difficult, sometimes unsatisfactory for the patient and an ophthalmologist. good glycemic control is still the most important Among all the methods of treating diabetes and its complication.

Glucose is known to be the main source of energy in organisms. Its metabolism in the cells involves redox reactions to produce energy by transport, storage or extraction of electrons. [33]. In the con- ditions of normal glycemic level, glucose undergoes the glycolysis pathway to produce ATP in the Kreb's cycle in the mitochondria, where electrons are being stored in form reduced form of Nicotinamide adenine dinucleotide (NADH) and flavin adenine dinucleotide (FADH2 [34]. ROS production takes place in mitochondria by two processes, one is the mitochondrial oxidative phosphorylation and the second NADH oxidase system (Nox). It had been described that over $95 \%$ of oxygen transformations take place in the mitochondria. Approximately $1-5 \%$ of these reactions result in ROS production. In the environment of hyperglycemia, increased level of ROS stabilizes hypoxia-inducible factor $1 \alpha$ (HIF-1 $\alpha$ ) leading to upregulation of angiogenic genes e.g. VEGF [35]. Data form the experiment had shown an increased level of VEGF in vitreous body of patients with proliferative diabetic retinopathy. Eye fundus examination revealed pathological neovascularization in retina, secondary to VEGF increased concentration [36].

Another study described the evidence for the correlation between oxidative stress and the severity of diabetic retinopathy. Retinal vessels' damage is caused by four major metabolic mechanismsincreases polyol pathway flux, activation of the protein kinase $\mathrm{C}(\mathrm{PKC})$ pathway, increased intracellular formation of advanced glycation end-proteins (AGEs) and expression of their receptors, and hexosamine activation pathway. All of these mechanisms have been positively correlated with increased oxidative stress and ROD overproduction [37]. On the other hand, obesity and dyslipidemia are different external factors leading to ROS overproduction, by fatty acids oxidation with NADPH oxidase. Experimental data showed that degeneration of retinal vessels could be reduced by antioxidant agents by activation of caspase- 3 and nuclear factor $-\kappa \mathrm{B}(\mathrm{NF}-\kappa \mathrm{B})$, which lead to the assumption that oxidative stress is one of the factors causing capillary apoptosis [38].

Longtime clinical observations suggest that decreasing oxidative stress may become part of DR treatment, as they delay or inhibit pathological metabolic pathways producing ROS [39].

\section{CONCLUSIONS}

It is proven that high oxidative stress damages retina, by the acceleration of photoreceptors and ganglion cells apoptosis. The denegation of retina, irrespective of the type, especially in macular dis- 
eases, causes vision impaired. In great majority of retinal diseases improvement prognosis is very poor. Oxidative stress agents, their influence on particular tissues, the oxidant-antioxidant balance is being still explored. Treatment is being aimed to cure the disease in its asymptomatic stage, to prevent vision impairment. Available data suggest that substances scavenging oxidative stress may be effective slowing down the progression of these degenerative ocular diseases. Therefore, inhibition of the metabolic pathways, producing a high concentration of ROS, may become protective for retinal cells. Currently, there are only cell or animal models studies proving beneficial use of antioxidants in the prevention of retinal damage. Development of the treatment for retinal degeneration still faces great challenges and further investigation is needed. However, predicted effects of antioxidant treatment are ambiguous, successful results of experimental studies lead straight to clinical use in human in the future.

\section{REFERENCES}

1. Siegfried CJ, Shui YB. Intraocular Oxygen and Antioxidant Status: New Insights on the Effect of Vitrectomy and Glaucoma Pathogenesis. Am J Ophthalmol. 2019; 203: 12-25, doi: 10.1016/j.ajo.2019.02.008, indexed in Pubmed: 30772349

2. Young IS, Woodside JV. Antioxidants in health and disease. J Clin Pathol. 2001; 54(3): 176-186, doi: 10.1136/jcp.54.3.176, indexed in Pubmed: 11253127.

3. Saccà SC, Izzotti A. Oxidative stress and glaucoma: injury in the anterior segment of the eye. Prog Brain Res. 2008; 173: 385-407, doi: 10.1016/S0079-6123(08)01127-8, indexed in Pubmed: 18929123.

4. Saccà SC, Roszkowska AM, lzzotti A. Environmental light and endogenous antioxidants as the main determinants of non-cancer ocular diseases. Mutat Res. 2013; 752(2): 153-171, doi: 10.1016/j. mrrev.2013.01.001, indexed in Pubmed: 23337404.

5. Bishop P. The biochemical structure of mammalian vitreous. Eye (Lond). 1996; 10 ( Pt 6): 664-670, doi: 10.1038/eye.1996.159, indexed in Pubmed: 9091361.

6. Kita T, Sakamoto T, Ishibashi T. Hyalocytes: Essential Vitreous Cells in Vitreoretinal Health and Disease. In: Vitreous. Springer, Berlin/ Heidelberg 2014: 151-164.

7. Kański J. Okulistyka kliniczna. Elsevier Urban \& Partner, Wrocław 2013: 716.

8. Sebag J. Posterior Vitreous Detachment. Ophthalmology. 2018; 125(9): 1384-1385, doi: 10.1016/j.ophtha.2018.05.018, indexed in Pubmed: 30143091.

9. Nuzzi R, Marchese A, Gulino GR, et al. Influence of posterior vitreous detachment and type of intraocular lens on lipid peroxidation in the human vitreous. Mol Vis. 2015; 21: 1106-1112, doi: 10.1038/ eye.2016.143, indexed in Pubmed: 26396488.

10. Ueno N, Sebag J, Hirokawa H, et al. Effects of visible-light irradiation on vitreous structure in the presence of a photosensitizer. Exp Eye Res. 1987; 44(6): 863-870, doi: 10.1016/s0014-4835(87)80048-9, indexed in Pubmed: 3653277.

11. Akiba J. [Photodynamically induced vitreous liquefaction in vivo]. Nippon Ganka Gakkai Zasshi. 1992; 96(6): 731-736, indexed in Pubmed: 1626474.

12. Berra A, Ferreira $S$, Stanga $P$, et al. Age-related antioxidant capacity of the vitreous and its possible relationship with simultaneous changes in photoreceptors, retinal pigment epithelium and Bruchs' membrane in human donors' eyes. Arch Gerontol Geriatr. 2002; 34(3): 371-377, doi: 10.1016/s0167-4943(02)00013-4, indexed in Pubmed: 14764337.

13. Goldberg H. Retinal Detachment. BMJ. 1959; 2(5144): 153-154, doi: 10.1136/bmj.2.5144.153-c, indexed in Pubmed: 24807890.

14. Bechrakis NE, Dimmer A. Rhegmatogene Netzhautablösung. Der Ophthalmologe. 2018; 115(2): 163-178, doi: 10.1007/s00347-017-0647-z.

15. Kański J, Jacek J. Okulistyka kliniczna. Elsevier Urban \& Partner, Wrocław 2013: 674-678.

16. Suzuki Y, Adachi K, Takanashi S, et al. Oxidative Stress in the Vitreous Fluid with Rhegmatogenous Retinal Detachment. J Clin Exp Ophthalmol. 2017; 6(5): 1-5, doi: 10.4172/2155-9570.1000682.

17. Maeno A, Suzuki Y, Adachi $K$, et al. Characterization of the biological antioxidant potential in the vitreous fluid from patients with rhegmatogenous retinal detachment. Acta Ophthalmol. 2016; 94(6): e515-e516, doi: 10.1111/aos.13002, indexed in Pubmed: 27008980.

18. She X, Lu X, Li T, et al. Inhibition of Mitochondrial Fission Preserves Photoreceptors after Retinal Detachment. Am J Pathol. 2018; 188(7): 1713-1722, doi: 10.1016/j.ajpath.2018.03.013, indexed in Pubmed: 29684364.

19. Roh Miln, Murakami Y, Thanos A, et al. Edaravone, an ROS scavenger, ameliorates photoreceptor cell death after experimental retinal detachment. Invest Ophthalmol Vis Sci. 2011; 52(6): 3825-3831, doi: 10.1167/iovs.10-6797, indexed in Pubmed: 21310909.

20. Lei H, Velez G, Cui J, et al. N-acetylcysteine suppresses retinal detachment in an experimental model of proliferative vitreoretinopathy. $\mathrm{Am}$ J Pathol. 2010; 177(1): 132-140, doi: 10.2353/ajpath.2010.090604, indexed in Pubmed: 20489144.

21. Kostrzewa B, Rojek A, Gabryś J. Starcze zwyrodnienie plamki żółtej - choroba cywilizacyjna XXI wieku. Acta Bio-Optica et Informatica Medica Inżynieria Biomedyczna. 2015; 21(2): 77-85.

22. Jonas JB, Cheung CM, Panda-Jonas S. Updates on the Epidemiology of Age-Related Macular Degeneration. Asia Pac J Ophthalmol (Phila). 2017; 6(6): 493-497, doi: 10.22608/AP0.2017251, indexed in Pubmed: 28906084.

23. Masuda T, Shimazawa M, Hara H. Retinal Diseases Associated with Oxidative Stress and the Effects of a Free Radical Scavenger (Edaravone). Oxid Med Cell Longev. 2017; 2017: 9208489, doi: 10.1155/2017/9208489, indexed in Pubmed: 28194256.

24. Golden TR, Hinerfeld DA, Melov S. Oxidative stress and aging: beyond correlation. Aging Cell. 2002; 1(2): 117-123, doi: 10.1046/j.14749728.2002.00015.x, indexed in Pubmed: 12882341.

25. Katz $M$, Robison W. What is lipofuscin? Defining characteristics and differentiation from other autofluorescent lysosomal storage bodies. Archives of Gerontology and Geriatrics. 2002; 34(3): 169-184, doi: 10.1016/s0167-4943(02)00005-5, indexed in Pubmed: 14764321.

26. Kaarniranta K, Pawlowska E, Szczepanska J, et al. Role of Mitochondrial DNA Damage in ROS-Mediated Pathogenesis of Age-Related Macular Degeneration (AMD). Int J Mol Sci. 2019; 20(10), doi: 10.3390/ ijms20102374, indexed in Pubmed: 31091656.

27. Domènech EB, Marfany G. The Relevance of Oxidative Stress in the Pathogenesis and Therapy of Retinal Dystrophies. Antioxidants (Basel). 2020; 9(4), doi: 10.3390/antiox9040347, indexed in Pubmed: 32340220.

28. Takayama K, Kaneko H, Kataoka K, et al. Nuclear Factor (ErythroidDerived)-Related Factor 2-Associated Retinal Pigment Epithelial Cell Protection under Blue Light-Induced Oxidative Stress. Oxid Med Cell Longev. 2016; 2016: 8694641, doi: 10.1155/2016/8694641, indexed in Pubmed: 27774118.

29. Kansanen E, Kuosmanen SM, Leinonen H, et al. The Keap1-Nrf2 pathway: Mechanisms of activation and dysregulation in cancer. Redox Biol. 2013; 1: 45-49, doi: 10.1016/j.redox.2012.10.001, indexed in Pubmed: 24024136.

30. Felszeghy S, Viiri J, Paterno JJ, et al. Loss of NRF-2 and PGC-1 genes leads to retinal pigment epithelium damage resembling dry age-related macular degeneration. Redox Biol. 2019; 20: 1-12, doi: 10.1016/j. redox.2018.09.011, indexed in Pubmed: 30253279.

31. Datta S, Cano M, Ebrahimi K, et al. The impact of oxidative stress and inflammation on RPE degeneration in non-neovascular AMD. Prog Retin Eye Res. 2017; 60: 201-218, doi: 10.1016/j.preteyeres.2017.03.002, indexed in Pubmed: 28336424. 
32. World Health Organization. Global status report on noncommunicable diseases 2014 (No. WHO/NMH/NVI/15.1). WHO, Geneva 2014.

33. Cecilia OM, José Alberto CG, José NP, et al. Oxidative Stress as the Main Target in Diabetic Retinopathy Pathophysiology. J Diabetes Res. 2019; 2019: 8562408, doi: 10.1155/2019/8562408, indexed in Pubmed: 31511825.

34. Yan LJ. Redox imbalance stress in diabetes mellitus: Role of the polyol pathway. Animal Model Exp Med. 2018; 1(1): 7-13, doi: 10.1002/ ame2.12001, indexed in Pubmed: 29863179.

35. Masuda T, Shimazawa M, Hara H. Retinal Diseases Associated with Oxidative Stress and the Effects of a Free Radical Scavenger (Edaravone). Oxid Med Cell Longev. 2017; 2017: 9208489, doi: 10.1155/2017/9208489, indexed in Pubmed: 28194256.
36. Izuta $H$, Matsunaga N, Shimazawa $M$, et al. Proliferative diabetic retinopathy and relations among antioxidant activity, oxidative stress, and VEGF in the vitreous body. Mol Vis. 2010; 16: 130-136, indexed in Pubmed: 20142849.

37. Kowluru RA, Chan PS. Oxidative stress and diabetic retinopathy. Exp Diabetes Res. 2007; 2007: 43603, doi: 10.1155/2007/43603, indexed in Pubmed: 17641741.

38. Kowluru RA, Koppolu P. Diabetes-induced activation of caspase-3 in retina: effect of antioxidant therapy. Free Radic Res. 2002; 36(9): 993-999, doi: $10.1080 / 1071576021000006572$, indexed in Pubmed: 12448825.

39. Williams M, Hogg RE, Chakravarthy U. Antioxidants and diabetic retinopathy. Curr Diab Rep. 2013; 13(4): 481-487, doi: 10.1007/ s11892-013-0384-x, indexed in Pubmed: 23649947. 